\title{
A cytogenetic study of the Caspian pony
}

\author{
H. Hatami-Monazah and R. V. Pandit \\ Department of Animal Biology, School of Veterinary Medicine, Shiraz University, Shiraz, Iran
}

\begin{abstract}
Summary. The group of Caspian ponies studied contained some animals with 65 chromosomes and others with 64 chromosomes. The morphology and G-banding pattern of the chromosomes resembled those of Equus caballus and E. przewalskii. The karyogram of animals with 65 chromosomes was identical to that of the cross between $E$. caballus and $E$. przewalskii. It is suggested that the Caspian pony is the product of natural hybridization between $E$. caballus and $E$. przewalskii. Low reproductive efficiency of the Caspian pony is suggested as the cause of decline in the population of these animals.
\end{abstract}

\section{Introduction}

In 1969, a miniature horse called the Caspian pony, was found in the northern part of Iran around the Caspian sea. The conformation of this pony is similar to that of the horse (Pl. 1, Fig. 1), and apart from the height there are only a few minor anatomical differences between this animal and Equus caballus (Firouz, 1971). Caspian ponies are short animals with bay, gray and chestnut as the most common colours. The head is short with a bulging forehead and small ears and the animal has oval feet. This pony has a graceful and perfectly proportioned body and is gentle, intelligent and very willing to work, thus making it very well suited as a riding horse for children.

A search for 3 years revealed that there are not more than 50 of these ponies left along the Caspian coast (Firouz, 1969). A group of about 20 ponies has been gathered together at this institution for scientific study. Apart from coat colour, animals in this group are phenotypically uniform.

In archaeological artefacts of Persia (Iran), such as seals, statuettes, reliefs and writings, similar horses are represented. Amongst these are small horses carved on the bas-reliefs in Persepolis, and on the trilingual seal of Darius the Great (500 B.c.), now in the British museum, two small ponies with slim legs and convex faces are shown pulling the royal chariot (Firouz, 1969; Afshar, 1972). Sculptures at Persepolis clearly indicate that these pony-like horses were gifts to the Persian Emperor by the Armenians who were subjects of the Persian empire during the 4th and 5th century B.C. (Sami, 1976). The archaeological evidence for the important role and possible historical continuity of a small horse from the pre-Achaemenian period to about the time of Islamic conquest in the 7th century A.D. has been discussed by Firouz (1971).

Chromosome analysis of the animals is clearly important and may help to elucidate the link between this pony and Equus caballus and also with other equids known from this area such as Equus przewalskii-poliakoff (Mohr, 1959). Carroll (1976), who had access to the preliminary results of this work, prematurely reported the chromosome complement of the Caspian pony as typical of Equus caballus, but the present report is of the analysis of more material.

\section{Material and Methods}

Heparinized blood samples were taken from 7 male and 10 female Caspian ponies and blood leucocytes were cultured by the short-term culture method (Moorhead, Nowell, Mellman, 
Battips \& Hungerford, 1960), but homologous serum instead of fetal calf serum was used in the medium (Pandit, 1978). The cells were arrested at metaphase with Colcemid (Ciba) added to the culture at a final concentration of $0.03 \mu \mathrm{g} / \mathrm{ml} 2 \mathrm{~h}$ before harvesting the cells. The cells were treated with hypotonic solution (Hanks B.S.S. + water, $1: 4 \mathrm{v} / \mathrm{v}$ ) for $20 \mathrm{~min}$ and fixed for an equal length of time in a mixture of methanol and acetic acid $(3: 1 \mathrm{v} / \mathrm{v})$, before spreading on clean pre-chilled slides. The slides were air dried and stained by the combination G-banding technique recommended by Parks \& Stenchever (1975). At least 25-30 spreads were examined from each slide and some metaphase figures were photographed with a Zeiss II photomicroscope.

\section{Results}

The Caspian ponies examined cytogenetically consisted of two groups of animals. One group was of animals possessing a modal number of 65 chromosomes while the other group showed 64 chromosomes. Out of the 17 animals analysed for chromosomes, 11 had 64 chromosomes while 6 had 65 chromosomes. There was no correlation between chromosome number and the sex of the animal or physical appearance. The 64 chromosomes consisted of 26 metacentric or submetacentric and 36 acrocentric autosomes and 2 sex chromosomes of which the $\mathrm{X}$ was a large submetacentric and the $\mathrm{Y}$ was a small acrocentric. The G-band pattern of these chromosomes indicated close resemblance to both E. caballus and E. przewalskii.

The animals with 65 chromosomes had 25 metacentric or submetacentric and 38 acrocentric autosomes and 2 sex chromosomes. The metacentric chromosomes could be arranged as 12 pairs with fairly well matching bands and arm lengths. The remaining chromosome was a more or less balanced metacentric with two major bands on one side and 2 major and 1 minor band on the other. According to its size this chromosome was assigned position number 10 as shown in the karyotype (Pl. 2, Figs 2 and 3). In the same group of animals, the 38 acrocentric chromosomes fell into 3 groups according to size. The chromosomes of positions 23 and 24 in the figure had a size and bands more or less identical to those of the two arms of the single chromosome at position 10 respectively.

In spite of free access to males, the conception rate in the Caspian females in this herd was low, never more than $40 \%$. Besides being summer breeders, the Caspian mares have problems in ovulation. The stallions showed a low sperm count and poor sperm motility, further contributing to the reproductive inefficiency of these animals.

\section{Discussion}

The conclusion of Carroll (1976) that the Caspian pony possesses 64 chromosomes was premature since data on ponies with 65 chromosomes were not available at that time. Subsequent analysis has revealed that a third of the ponies have 65 chromosomes.

In the Equidae odd chromosomes are present in hybrids such as mules and hinnies, but these animals are infertile because of their inability to achieve homologous pairing during meiosis (Wodsedalek, 1916; Makino, 1955; Taylor \& Short, 1975). As a result, $F_{1}$ progeny between horse and donkey are unable to start a continuous hybrid line of animals. The Caspian pony, although an inefficient breeder, has a long history of continuous breeding. A considerable number of animals in our random sample of the population possess a modal number of chromosomes between that of $E$. caballus $(2 \mathrm{n}=64)$ and $E$. przewalskii $(2 \mathrm{n}=66)$. The morphology and G-bands of the chromosomes of the Caspian pony resemble those of $E$. caballus as well as E. przewalskii. These facts, together with the historical background (see 'Introduction') suggest that the Caspian pony could be the outcome of natural hybridization 
PLATE 1

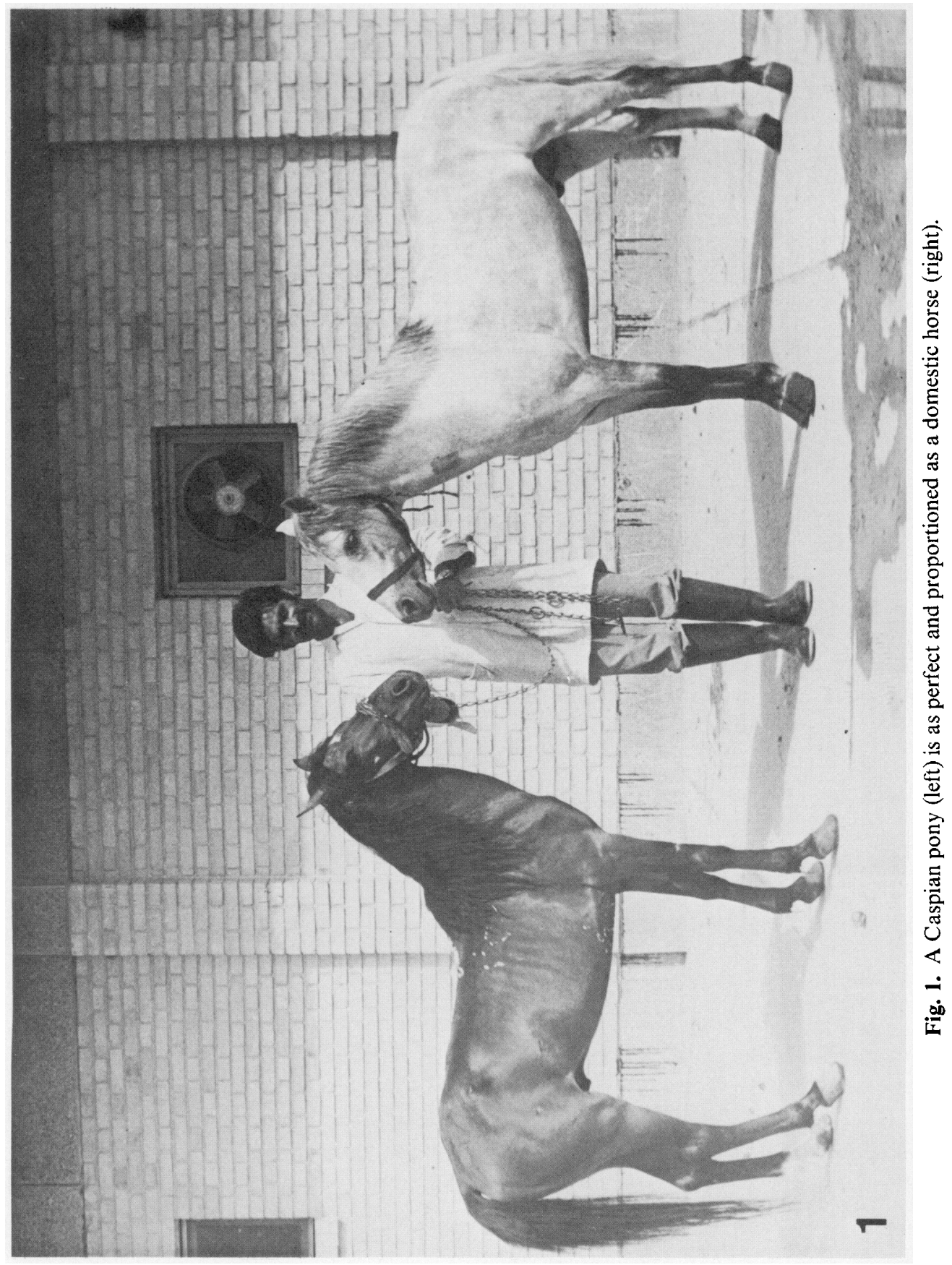

(Facing p. 332) 
PLATE 2
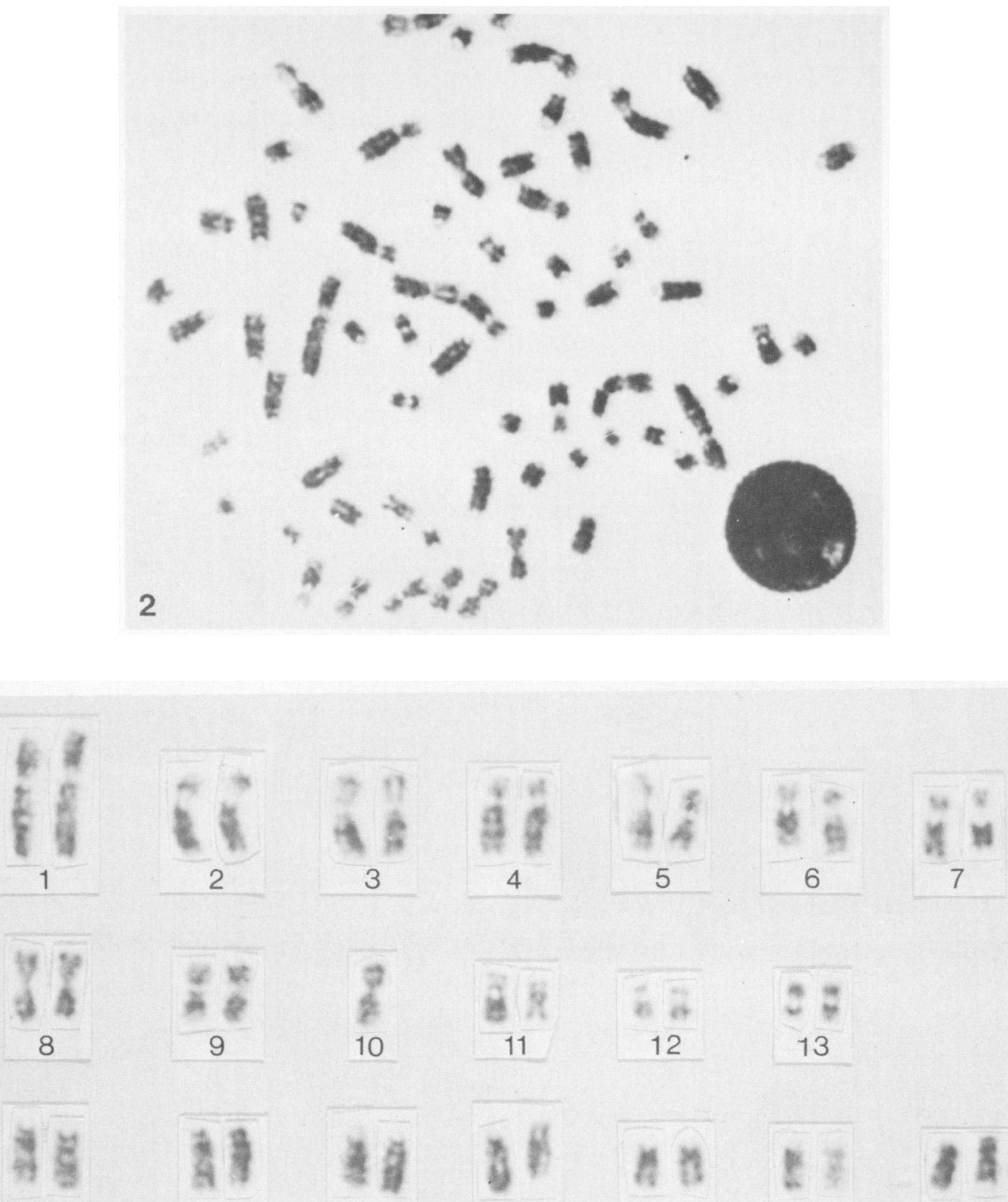

14
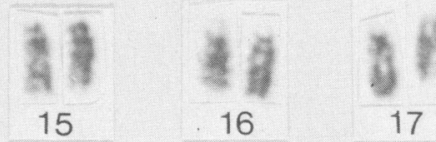

18

19

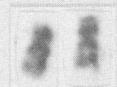

\section{5}

. 16
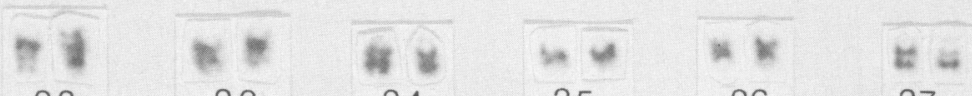

21

$$
22
$$

23

24

25

26
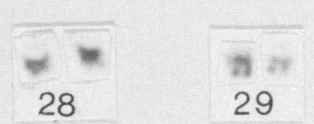

29
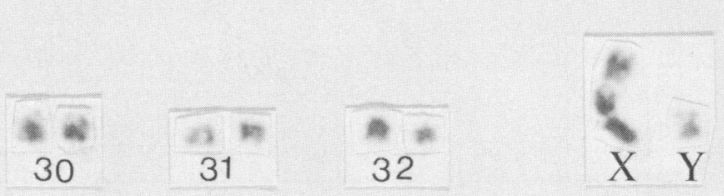

3

Figs 2 and 3. A metaphase plate (Fig. 2) and a karyotype (Fig. 3) of a Caspian pony with 65 chromosomes. One odd metacentric chromosome is seen at position 10 in Fig. 3. 
between $E$. caballus and $E$. przewalskii. It has been suggested that 4 acrocentric chromosomes have been replaced by 2 metacentric chromosomes in $E$. caballus through a process of centric fusion (Benirschke, Malouf, Low \& Heck, 1965; Benirschke \& Malouf, 1967), and a fertile cross between $E$. caballus and $E$. przewalskii under natural conditions is therefore likely. Such a cross in a zoo has been reported by Koulischer \& Frenchkop (1966). Chandley, Short \& Allen (1975) have also studied an experimental cross between $E$. caballus and $E$. przewalskii which was fertile and the $F_{2}$ progeny of this experiment had chromosome numbers of $2 n=64$ and $2 n=65$. The karyotype of the hybrid published by Chandley et al. (1975) is very similar to the karyotype of Caspian ponies with 65 chromosomes. This is additional evidence to support our hypothesis that the Caspian pony is the product of natural breeding between $E$. caballus and E. przewalskii. We suggest that the $F_{1}$ of such crosses uniformly had 65 chromosomes, followed by random distribution into populations with 64, 65 and 66 chromosomes, two of which were found in the present study on a very small sample of the population.

Parallel studies on reproductive behaviour of Caspian ponies at this institution have shown that although these animals are fertile, their breeding efficiency is low because of problems in spermatogenesis and ovulation. Results of studies on reproduction in these animals, still in progress, will be reported separately.

We thank the Shiraz University for facilities provided, Mrs E. Holmes and Miss S. Ghaibparvar for technical help and Mr Hadian for photographs.

\section{References}

Afshar, A. (1972) Rare breeds of domestic animals. Vet. Rec. 90, 20.

Benirschke, K. \& Malouf, N. (1967) Chromosome studies of Equidae. Equus 1, 253-284.

Benirschke, K., Malouf, N., Low, R.J. \& Heck, H. (1965) Chromosome complement: differences between $E$. caballus and E. przewalskii-poliakoff. Science, N.Y. 148, 382-383.

Carroll, R.E. (1976) Equine practitioner: Iran's Caspian horse. Modern Vet. Practice 57, 1027-1029.

Chandley, A.C., Short, R.V. \& Allen, W.R. (1975) Cytogenetic studies of three equine hybrids. $J$. Reprod. Fert., Suppl. 23, 365-370.

Firouz, L. (1969) Conservation of a domestic breed. Biol. Conservation 1, 1-2.

Firouz, L. (1971) Osteological and historical implication of the Caspian pony to early domestication in Iran. Proc. 3rd Int. Congr. Agricultural Museum, Budapest, 1-5.

Koulischer, L. \& Frechkop, S. (1966) Chromosome complement; a fertile hybrid between $E$. przewalskii and $E$. caballus. Science, N.Y. 151, 93-95.
Makino, S. (1955) Notes on the cytological feature of male sterility in the mule. Experientia 11, 224-226.

Mohr, E. (1959) Das Urwildpferd, Ziemsen, Wittenberg. Quoted by Benirschke et al. (1965).

Moorhead, D.A., Norwell, P.C., Mellman, W.J., Battips, D.W. \& Hungerford, D.A. (1960) Chromosome preparation of leucocytes cultured from human peripheral blood. Expl Cell Res. 20, 613-616.

Pandit, R.V. (1978) Use of homologous plasma for short term lymphocyte culture. Mammalian Chromosome Newsletter, 19, 386.

Parks, K.J. \& Stenchever, M.S. (1975) Combination Gbanding technique. Mammalian Chromosome Newsletter, 16, 27-29.

Sami, A. (1976) In Persepolis-Takht-I-Jamshid, 9th edn, 31 pp. Musavi Printing Office, Shiraz.

Taylor, M.J. \& Short, R.V. (1975) Development of the germ cells in the ovary of the mule and hinny. $J$. Reprod. Fert. 32, $441-445$.

Wodsedalek, J.E. (1916) Biol. Bull. 30, 1-57. Quoted by Chandley et al. (1975). 